\title{
Internationa国際会議案内 2002年3月～4月開催予定の国際会議
}

\section{3月4日〜 7 日}

COMDEX 2002 Conference and exposition.

場 所 : Chicago, IL (USA)

問合せ : Telefax: +1(781)449-2674

E-mail: register@key3media.com

http://www.key 3 media.com/comdex/ chicago2002/

\section{3月5日～8日}

2002 Annual meeting of the Association for Information and Image Management International (AIIM)

主 催 : Association for Information and Image Management (AIIM), Silver Spring, MD (USA)

場 所：San Francisco, CA (USA)

問合せ：AIIM International 1100, Wayne Avenue Suite 1100, Silver Spring, MD 20910, USA

Telefax: +1 (301) 5872711

http://www.aiim.org

\section{3月11日～12日}

2002 EUSIDIC spring meeting: E-content - Divide or rule.

主 催: European Association of Information Services (EUSIDIC)

場 所：Paris (France)

問合せ : EUSIDIC Secretariat, c/o Fachinformationszentrum Karlsruhe Frau Ute Jenschke Hermann-von-Helmholtz-Platz 1 D76344 Eggenstein-Leopoldshafen, Germany

Telefax: +49(0)7247/808-114

E-mail: eusidic@fiz-karlsruhe.de http://www.eusidic.org

\section{3月11日〜15日}

QWE2002: 5. Annual international Internet and software quality week Europe - Internet Now!, with exhibit.

主＼cjkstart催：Software Research Inst., San Francisco, CA (USA)

場 所：Brussels (Belgium)

問合せ：SR/Institute, Rita Bral, 901 Minnesota Street San

Francisco, CA 94107, USA

Telefax: +1(415)550-3030

E-mail: qw@soft.com

http://www.qualityweek.com/QWE2002/

\section{3月18日〜23日}

SITE 2002: 13. Annual international conference of the Society for Information Technology and Teacher Education (SITE).

共 催：Vanderbilt Univ., Nashville, TN (USA); Association for the Advancement of Computing in Education (AACE), Norfolk, VA (USA); Society for Information

場 所：Nashville, TN (USA)

問合せ：SITE/AACE P.O. Box: 3728 Norfolk, VA 23514, USA

Telefax: phone: +1(757) 623-7588

E-mail: conf@aace.org

http://www.aace.org/conf/site/

\section{3月18日〜20日}

Internet Librarian International 2002: International Internet conference and exhibition for librarians and information managers, with exhibition.

場 所: London (UK)

問合せ：Rubicon Communications Ltd. Oxford Centre for Innovation Mill Street, Oxford OX2 0JX, UK Telefax: $+44(0) 1865204950$

E-mail: info@internet-librarian.com http://www.internet-librarian.com/

\section{3月20日〜22日}

IT\&ILit2002: 1. International conference on IT and information literacy.

主 催：Glasgow Caledonian Univ. (UK); Strathclyde Univ., Glasgow (UK)

場 所 : Glasgow (UK)

問合せ : IT and ILit 2002 Secretariat IT Education Unit University of Glasgow Glasgow G12 8QQ, UK Telefax: +44(0)141-303-2602 E-mail: IT\&ILit2002@gla.ac.uk http://www.iteu.gla.ac.uk/IT\&ILit2002/IE/ index.html

\section{3月21日〜22日}

11. Internationale Fachkonferenz der Deutschen Gesellschaft fuer Informationswissenschaft und -praxis e.V. (DGI) und 
der Schweizerischen Vereinigung fuer Dokumentation (SVD-ASD): Business Intelligence - Neue Konzepte, Methoden und Instrumente.

11. International technical conference of Deutsche Gesellschaft fuer Informationswissenschaft und -praxis e.V. (DGI) and Schweizerische Vereinigung fuer Dokumentation (SVD-ASD): Business intelligence new concepts, methods and tools.

主 催: Deutsche Gesellschaft fuer Informationswissenschaft und Informationspraxis (DGI), Frankfurt am Main (Germany); Schweizerische Vereinigung fuer Dokumentation (SVD), Bern

場 所：Heidelberg (Germany)

問合せ : Deutsche Gesellschaft fuer Informationswissenschaft und Informationspraxis

Christine Fisch Ostbahnhofstr. 13 D-60314 Frankfurt am Main, Germany

Telefax: $+49(0) 69 / 4909096$

E-mail: fisch@dgi-info.de

http://www.dgi-online.org/

\section{4月3日〜6日}

ICEIS 2002: 4. International conference on enterprise information systems.

主 催：Universidad de Castilla-La Mancha (UCLM), Ciudad Real (Spain); Escola Superior de Tecnologia, Setubal (Portugal). Dept. de Sistemas e Informatica

場 所：Ciudad Real (Spain)

問合せ：Universidad de Castilla-La Mancha E.S. Informatica Ciudad Real, Spain

Telefax: +34926295354

E-mail: secretariat@iceis.org http://www.iceis.org/

\section{4月4日〜 6日}

CATA-2002: 17. International conference on computers and their applications.

主 催 : International Society for Computers and Their Applications (ISCA), Cary, NC (USA)

場 所：San Francisco, CA (USA)

問合せ：Southern Polytechnic State University ChichCheng Hung 1100 South Marietta Parkway Marietta, GA 30060, USA

Telefax: Phone: +1(770)528-3574

E-mail: chung@spsu.edu

\section{4月8日〜 10日}

CO2002: International symposium on combinatorial optimization.
場 所：Paris (France)

問合せ：LIP6-Universite Paris 6-Case 169 Philippe Chretienne 4, Place Jussieu Paris Cedex 05 F75252, France

E-mail: co2002@lip6.fr http://www-poleia.lip6.fr/ safia/CO2002/

\section{4月8日〜 10日}

Soft-Ware 2002: 1. International conference on computing in an imperfect world: Face life as it is, not as you would like it to be.

場 所：Belfast (UK)

問合せ ：E-mail: soft-ware@inf.ulst.ac.uk http://www.infc.ulst.ac.uk/informatics/ events.soft-ware/

\section{4月8日〜10日}

ITCC 2002: International conference on information technology-Coding and computing.

主 催：Institute of Electrical and Electronics Engineers, Inc., Washington, DC (USA). Computer Society

場 所：Las Vegas, NV (USA)

問合せ：University of Nevada Howard R. Hughes College of Engineering, Prof. Shahram Latifi, 4505 Maryland Parkway Las Vegas, NV 89154-4026, USA

Telefax: +1(702)895-4075

E-mail: latifi@ee.unlv.edu http://www.cs.clemson.edu/ srimani/itcc2002/ cfp.html

\section{4月11日〜13日}

2. SIAM International conference on data mining.

主 催 : Society for Industrial and Applied Mathematics (SIAM), Philadelphia, PA (USA)

場 所: Arlington, VA (USA)

問合せ：Society for Industrial and Applied Mathematics 3600 University City Science Center Philadelphia, PA 19104-2688, USA

Telefax: +1(215) 386-7999

E-mail: service@siam.org http://www.siam.org/

\section{4月15日〜17日}

EUROMEDIA 2002: WEBTEC, MEDIATEC, COMTEC, APTEC, and ETEC.

主 催: Society for Modeling and Simulation International-SCS European Simulation Office, Gent (Belgium)

場 所：Modena (Italy) 
問合せ : SCS European Simulation Office University of Gent Philippe Geril Coupure Links 653 B-9000 Gent, Belgium

Telefax: +32(9) 2234941

E-mail: philippe.geril@rug.ac.be

http://biomath.rug.ac.be/ scs/conf/euromd2002/

\section{十月17日〜20日}

itx: Messe fuer Informationstechnologien, Internet, Multimedia und Office-Solutions.

itx: Fair on information technologies, Internet, multimedia and office solutions.

主 催 : Messe Stuttgart International (Germany)

場 所: Stuttgart (Germany)

問合せ：Messe Stuttgart International Am Kochenhof 16 D-70192 Stuttgart, Germany

\section{จ 4月21日〜26日}

34. IUVSTA workshop: XPS - from spectra to results towards an expert system.

主 催: International Union for Vacuum Science, Technique and Applications, Brussels (Belgium)

場 所: Saint-Malo (France)

問合せ : National Inst. of Standards and Technology Cedric Powell Route 270 and Quince Orchard Rd. Gaithersburg, MD 20899-0001, USA

E-mail: cedric.powell@nist.gov

\section{4月21日～24日}

ICCS 2002: 2. International conference on computational science.

主 催 : Society for Industrial and Applied Mathematics (SIAM), Philadelphia, PA (USA); HPCN-Europe Foundation

場 所: Amsterdam (Netherlands)

問合せ：Universiteit van Amsterdam ICCS 2002 Peter Sloot Kruislaan 403 NL-1098 SJ Amsterdam, Netherlands

Telefax: +31-20-5257419

E-mail: iccs@science.uva.nl

http://www.science.uva.nl/events/ICCS2002/

४ 4月22日 25日

KR2002: 8. International conference on principles of knowlegde representation and reasoning.

場 所: Toulouse (France)

問合せ：Universite Paul Sabatie Inst. de Recherche en Informatique Andreas Herzig Toulouse, France
E-mail: herzig@irit.fr

http://www.kr.org/kr/kr02/

\section{4月22日～26日}

Info 2002: Congreso internacional de informacion Informacion conocimiento y sociedad - Retos de una nueva era.

Info 2002: International congress of information Information, knowledges and society - Challenges of new era.

主 催: Academia de Ciencias de Cuba, La Habana (Cuba). Inst. de Documentacion e Informacion Cientifica y Tecnica (IDICT)

場 所: La Habana (Cuba)

問合せ : IDICT Lic. Nicolas Garriga Mendez Apartado 2019 La Habana 10200, Cuba

Telefax: (537) 338237

E-mail: info@idict.cu

http://www.idict.cu/info2002/venglish.htm

\section{4月23日〜26日}

Interflow 2002: Anwenderkongress fuer dokumentenbasierte Technologien.

Interflow 2002: User congress for document based technologies.

主 催 : Institute for International Research $\mathrm{GmbH}$ und Co., Sulzbach (Germany)

場 所: Wiesbaden (Germany)

問合せ : IIR Deutschland GmbH Anna Katharina Caspary Otto-Volger-Strasse 17 D-65843 Sulzbach/Ts., Germany

Telefax: +49(0)6196/585-485

E-mail: kcaspary@iir.de http://www.interflow.de

\section{4月24日〜26日}

MIS 2002: 3. International conference on management information systems, incorporating GIS and remote sensing.

主 催: Wessex Inst. of Technology (WIT), Southampton (UK)

場 所: Chalkidiki (Greece)

問合せ : Wessex Inst. of Technology Gabriella Cossutta Ashurst Lodge, Ashurst Southampton SO40 7AA, UK

Telefax: $+44(0) 1703292853$

E-mail: gcossutta@wessex.ac.uk http://www.wessex.ac.uk/conferences/2002/ mis02/ 